Int. J. Contemp. Math. Sciences, Vol. 2, 2007, no. 8, 357 - 372

\title{
Subclasses of p-Valent and Prestarlike Functions
}

\author{
M. K. Aouf \\ Dept. of Math., Faculty of Science \\ Univ. of Mansoura, Mansoura 35516, Egypt \\ mkaouf127@yahoo.com
}

H. Silverman

Dept. of Math., Univ. of Charleston

Charleston, South Carolina 29424, USA

silvermanh@cofc.edu

\begin{abstract}
The object of the present paper is to investigate coefficients for functions belonging to the subclasses $R^{p}[\alpha, \beta]$ and $C^{p}[\alpha, \beta]$ of p-valent $\alpha$ prestarlike functions of order $\beta$ with negative coefficients. We obtain closure theorems, integral operators, radius of starlikeness and distortion theorems for functions belonging to the classes $R^{p}[\alpha, \beta]$ and $C^{p}[\alpha, \beta]$. We also obtain several results for the modified Hadamard products of functions belonging to the classes $R^{p}[\alpha, \beta]$ and $C^{p}[\alpha, \beta]$.
\end{abstract}

\section{Mathematics Subject Classification: 30C45}

Keywords: p-Valent, prestarlike functions, closure theorems, modified Hadamard products

\section{Introduction}

Let $A(p)$ denote the class of functions of the form:

$$
f(z)=z^{p}+\sum_{n=1}^{\infty} a_{p+n} z^{p+n}(p \in N=\{1,2, \ldots\})
$$

which are analytic and p-valent in the unit disc $U=\{z:|z|<1\}$. A function $f(z) \in A(p)$ is called p-valent starlike of order $\alpha(0 \leq \alpha<p)$ if $f(z)$ satisfies the conditions 


$$
\operatorname{Re}\left\{\frac{z f^{\prime}(z)}{f(z)}\right\}>\alpha \quad(z \in U)
$$

and

$$
\int_{0}^{2 \pi} \operatorname{Re}\left\{\frac{z f^{\prime}(z)}{f(z)}\right\} d \theta=2 \pi p(z \in U) .
$$

We denote by $S^{*}(p, \alpha)$ the class of p-valent starlike functions of order $\alpha$. The class $S^{*}(p, \alpha)$ was introduced by Patil and Thakare [8].

The function

$$
s_{\alpha}^{p}(z)=\frac{z^{p}}{(1-z)^{2(p-\alpha)}}(0 \leq \alpha<p ; p \in N)
$$

is the familiar extremal function for the class $S^{*}(p, \alpha)$. Setting

$$
G^{p}(\alpha, n)=\frac{\prod_{m=2}^{n}[2(p-\alpha)+m-2]}{(n-1) !}(n \in N /\{1\}, 0 \leq \alpha<p),
$$

$s_{\alpha}^{p}(z)$ can be written in the form:

$$
s_{\alpha}^{p}(z)=z^{p}+\sum_{n=1}^{\infty} G^{p}(\alpha, n+1) z^{p+n} .
$$

Clearly, $s_{\alpha}^{p}(z) \in S^{*}(p, \alpha)$ and $G^{p}(\alpha, n+1)$ is a decreasing function in $\alpha(0 \leq$ $\left.\alpha \leq \frac{2 p-1}{2} ; p \in N\right)$ and satisfies

$$
\lim _{n \rightarrow \infty} G^{p}(\alpha, n+1)= \begin{cases}\infty & \left(\alpha<\frac{2 p-1}{2}\right) \\ 1 & \left(\alpha=\frac{2 p-1}{2}\right) \\ 0 & \left(\alpha>\frac{2 p-1}{2}\right) .\end{cases}
$$

Let $(f * g)(z)$ denote the Hadamard product (or convolution) of the functions $f(z)$ and $g(z)$, that is, if $f(z)$ is given by (1.1) and $g(z)$ is given by

$$
g(z)=z^{p}+\sum_{n=1}^{\infty} b_{p+n} z^{p+n}
$$

then 


$$
(f * g)(z)=z^{p}+\sum_{n=1}^{\infty} a_{p+n} b_{p+n} z^{p+n} .
$$

A function $f(z) \in A(p)$ is said to be p-valent $\alpha$-prestarlike function of order $\beta(0 \leq \alpha<p ; 0 \leq \beta<p ; p \in N)$ if

$$
\left(f * s_{\alpha}^{p}\right)(z) \in S^{*}(p, \beta),
$$

where $s_{\alpha}^{p}(z)$ is defined by (1.4). We denote by $R^{p}(\alpha, \beta)$ the class of all pvalent $\alpha$-prestarlike functions of order $\beta$. For $\alpha=\frac{2 p-1}{2} ; 0 \leq \beta<p ; p \in$ $N, R^{p}\left(\frac{2 p-1}{2}, \beta\right)=S^{*}(p, \beta)$. Further let $C^{p}(\alpha, \beta)$ be the subclass of $A(p)$ consisting of functions $f(z)$ satisfying

$$
f(z) \in C^{p}(\alpha, \beta) \text { if and only if } \frac{z f^{\prime}(z)}{p} \in R^{p}(\alpha, \beta) .
$$

We note that:

(i) $R^{p}(\alpha, \alpha)=R^{p}(\alpha)(0 \leq \alpha<p ; p \in N)$, the class of p-valent prestarlike functions of order $\alpha$, was studied by Kumar and Reddy [3] and Shenen et al. [13];

(ii) $R^{1}(\alpha, \beta)=R_{\alpha, \beta}(0 \leq \alpha<1 ; 0 \leq \beta<1)$, the class of $\alpha$-prestarlike functions of order $\beta$, was introduced by Sheil-Small et al. [12];

(iii) $R^{1}(\alpha, \alpha)=R_{\alpha}(0 \leq \alpha<1)$, the class of prestarlike functions of order $\alpha$, was introduced by Ruscheweyh [10];

(iv) $C^{1}(\alpha, \beta)=C(\alpha, \beta)(0 \leq \alpha<1 ; 0 \leq \beta<1)$, the subclass of $A(1)=A$ consisting of functions $f(z) \in A$ satisfying $z f^{\prime}(z) \in R^{1}(\alpha, \beta)=R(\alpha, \beta)$, was introduced by Owa and Uralegaddi [7].

Denoting by $T(p)$ the subclass of $A(p)$ consisting of functions of the form:

$$
f(z)=z^{p}-\sum_{n=1}^{\infty} a_{p+n} z^{p+n}\left(a_{p+n} \geq 0 ; p \in N\right) .
$$

We denote by $S^{*}[p, \beta], R^{p}[\alpha, \beta]$ and $C^{p}[\alpha, \beta]$ the classes obtained by taking intersections, respectively, of the classes $S^{*}(p, \beta), R^{p}(\alpha, \beta)$ and $C^{p}(\alpha, \beta)$ with the class $T(p)$. Thus, we have

$$
\begin{gathered}
S^{*}[p, \beta]=S^{*}(p, \beta) \cap T(p), \\
R^{p}[\alpha, \beta]=R^{p}(\alpha, \beta) \cap T(p),
\end{gathered}
$$

and

$$
C^{p}[\alpha, \beta]=C^{p}(\alpha, \beta) \cap T(p)
$$


The class $S^{*}[p, \beta]$ was studied by Owa [5].

It follows from (1.13) and (1.14) that

$$
f(z) \in C^{p}[\alpha, \beta] \text { if and only if } \frac{z f^{\prime}(z)}{p} \in R^{p}[\alpha, \beta] .
$$

Also we note that, by specializing the parameters $\alpha, \beta$ and $p$, we obtain the following subclasses studied by various authors:

(i) $R^{p}[p \alpha, p \alpha]=R^{p}[\alpha](0 \leq \alpha<1 ; p \in N)$ (Kumar and Reddy [3]);

(ii) $R^{1}[\alpha, \alpha]=R[\alpha](0 \leq \alpha<1)$ (Silverman and Silvia [14] and Owa and Al-Bassam [6]);

(iii) $R^{1}[\alpha, \beta]=R[\alpha, \beta](0 \leq \alpha<1,0 \leq \beta<1)$ (Silverman and Silvia [15], Uralegaddi and Sarangi [17], Aouf and Salagean [2], Aouf et al. [1], Srivastava and Aouf [16] and Rania and Srivastava [9]).

In the present paper we investigate coefficients for functions belonging to the subclasses $R^{p}[\alpha, \beta]$ and $C^{p}[\alpha, \beta]$ of p-valent $\alpha$-prestarlike functions of order $\beta$ with negative coefficients. We obtain closure theorems, integral operators, radii of starlikeness and convexity and distortion theorems for functions belonging to the classes $R^{p}[\alpha, \beta]$ and $C^{p}[\alpha, \beta]$. We also obtain several results for the modified Hadamard products of functions belonging to the classes $R^{p}[\alpha, \beta]$ and $C^{p}[\alpha, \beta]$.

\section{Coefficient inequalities}

Theorem 1 . Let the function $f(z)$ be defined by (1.11). Then $f(z)$ is in the class $R^{p}[\alpha, \beta]$ if and only if

$$
\sum_{n=1}^{\infty}(n+p-\beta) G^{p}(\alpha, n+1) a_{p+n} \leq(p-\beta) .
$$

Proof. It is known [5] that a necessary and sufficient condition for $g(z)=$ $z^{p}-\sum_{n=1}^{\infty} b_{p+n} z^{p+n}\left(b_{p+n} \geq 0\right)$ to be in the class $S^{*}[p, \beta]$ is that

$$
\sum_{n=1}^{\infty}(n+p-\beta) b_{p+n} \leq(p-\beta) .
$$

Since

$$
\left(f * s_{\alpha}^{p}\right)(z)=z^{p}-\sum_{n=1}^{\infty} G^{p}(\alpha, n+1) a_{p+n} z^{p+n},
$$

where $s_{\alpha}^{p}(z)$ is given by (1.4), the result follows. 
Corollary 2 .If $f(z)$ is in the class $R^{p}[\alpha, \beta]$, then

$$
a_{p+n} \leq \frac{(p-\beta)}{(n+p-\beta) G^{p}(\alpha, n+1)}(p, n \in N),
$$

with equality for

$$
f(z)=z^{p}-\frac{(p-\beta)}{(n+p-\beta) G^{p}(\alpha, n+1)} z^{p+n}(p, n \in N) .
$$

In view of (1.15), Theorem 1 yields the following necessary and sufficient condition for the class $C^{p}[\alpha, \beta]$.

Theorem 3 . The function $f(z)$, defined by (1.11), is in the class $C^{p}[\alpha, \beta]$ if and only if

$$
\sum_{n=1}^{\infty}\left(\frac{p+n}{p}\right)(n+p-\beta) G^{p}(\alpha, n+1) a_{p+n} \leq(p-\beta) .
$$

\section{Extreme points}

From Theorem 1 and Theorem 2, we see that both $R^{p}[\alpha, \beta]$ and $C^{p}[\alpha, \beta]$ are closed under convex linear combinations, which enables us to determine the extreme points for these classes.

Theorem 4 . Let

$$
f_{p}(z)=z^{p}
$$

and

$$
f_{p+n}(z)=z^{p}-\frac{(p-\beta)}{(n+p-\beta) G^{p}(\alpha, n+1)} z^{p+n}(p, n \in N) .
$$

Then $f(z) \in R^{p}[\alpha, \beta]$ if and only if it can be expressed in the form

$$
f(z)=\sum_{n=0}^{\infty} \mu_{p+n} f_{p+n}(z)
$$

where $\mu_{p+n} \geq 0$ and $\sum_{n=0}^{\infty} \mu_{p+n}=1$.

Proof. Suppose that 


$$
\begin{aligned}
f(z) & =\sum_{n=0}^{\infty} \mu_{p+n} f_{p+n}(z) \\
& =z^{p}-\sum_{n=1}^{\infty} \frac{(p-\beta)}{(n+p-\beta) G^{p}(\alpha, n+1)} \mu_{p+n} z^{p+n} .
\end{aligned}
$$

Then it follows that

$$
\begin{aligned}
& \sum_{n=1}^{\infty} \frac{(n+p-\beta) G^{p}(\alpha, n+1)}{(p-\beta)} \cdot \frac{(p-\beta)}{(n+p-\beta) G^{p}(\alpha, n+1)} \mu_{p+n} \\
= & \sum_{n=1}^{\infty} \mu_{p+n}=1-\mu_{p} \leq 1 .
\end{aligned}
$$

Therefore, by Theorem $1, f(z) \in R^{p}[\alpha, \beta]$.

Conversely, assume that the function $f(z)$ defined by (1.11) belongs to the class $R^{p}[\alpha, \beta]$. Then

$$
a_{p+n} \leq \frac{(p-\beta)}{(n+p-\beta) G^{p}(\alpha, n+1)}(p, n \in N) .
$$

Setting

$$
\mu_{p+n}=\frac{(n+p-\beta) G^{p}(\alpha, n+1)}{(p-\beta)}(p, n \in N)
$$

and

$$
\mu_{p}=1-\sum_{n=1}^{\infty} \mu_{p+n},
$$

we see that $f(z)$ can be expressed in the form (3.3). This completes the proof of Theorem 3 .

Corollary 5 . The extreme points of the class $R^{p}[\alpha, \beta]$ are the functions $f_{p}(z)=z^{p}$ and

$$
f_{p+n}(z)=z^{p}-\frac{(p-\beta)}{(n+p-\beta) G^{p}(\alpha, n+1)} z^{p+n}(p, n \in N) .
$$

Similarly, we have

Theorem 6 .Let 


$$
f_{p}(z)=z^{p}
$$

and

$$
f_{p+n}(z)=z^{p}-\frac{(p-\beta)}{\left(\frac{p+n}{p}\right)(n+p-\beta) G^{p}(\alpha, n+1)} z^{p+n}(p, n \in N) .
$$

Then $f(z) \in C^{p}[\alpha, \beta]$ if and only if it can be expressed in the form

$$
f(z)=\sum_{n=0}^{\infty} \mu_{p+n} f_{p+n}(z)
$$

where $\mu_{p+n} \geq 0$ and $\sum_{n=0}^{\infty} \mu_{p+n}=1$.

Corollary 7 . The extreme points of the class $C^{p}[\alpha, \beta]$ are the functions $f_{p}(z)=$ $z^{p}$ and

$$
f_{p+n}(z)=z^{p}-\frac{(p-\beta)}{\left(\frac{p+n}{p}\right)(n+p-\beta) G^{p}(\alpha, n+1)} z^{p+n}(p, n \in N) .
$$

\section{Distortion theorems}

In view of Theorems 3 and 4, we will obtain distortion theorems for the classes $R^{p}[\alpha, \beta]$ and $C^{p}[\alpha, \beta]$.

Lemma 8 . For $0 \leq \alpha \leq \frac{2 p-1}{2}, 0 \leq \beta<p$ and $p \in N$, then $(n+p-$ $\beta) G^{p}(\alpha, n+1)$ is an increasing function of $n$, where $G^{p}(\alpha, n+1)$ is defined by (1.5).

Proof. . Let $K(\alpha, \beta, n, p)=(n+p-\beta) G^{p}(\alpha, n+1)$. Since,

$$
G^{p}(\alpha, n+2)=\frac{2 p+n-2 \alpha}{n+1} G^{p}(\alpha, n+1),
$$

we can see that $K(\alpha, \beta, n+1, p) \geq K(\alpha, \beta, n, p)$ if and only if

$$
2(p-\alpha)(n+1+p-\beta)-(p-\beta) \geq 0,
$$

for $0 \leq \alpha \leq \frac{2 p-1}{2}, 0 \leq \beta<p$ which holds for $p \in N$. This completes the proof of Lemma 1.

In the remainder of this section, we assume that $f(z)$ is defined by (1.11), $0 \leq \alpha \leq \frac{2 p-1}{2}, 0 \leq \beta<p$ and $p$ in $N$. 
Theorem 9 . If $f(z)$ is in the class $R^{p}[\alpha, \beta]$, then

$$
\begin{gathered}
|z|^{p}-\frac{(p-\beta)}{2(1+p-\beta)(p-\alpha)}|z|^{p+1} \leq|f(z)| \leq|z|^{p}+\frac{(p-\beta)}{2(1+p-\beta)(p-\alpha)}|z|^{p+1} \\
(z \in U)
\end{gathered}
$$

Equality holds for the function $f_{p+1}(z)$ given by

$$
f_{p+1}(z)=z^{p}-\frac{(p-\beta)}{2(1+p-\beta)(p-\alpha)} z^{p+1}(z \in U) .
$$

Proof. By virtue of Theorem 3, we note that

$$
|z|^{p}-\max _{n \in N} \frac{(p-\beta)}{(n+p-\beta) G^{p}(\alpha, n+1)}|z|^{p+n} \leq|f(z)| \leq|z|^{p}+\max _{n \in N} \frac{(p-\beta)}{(n+p-\beta) G^{p}(\alpha, n+1)}|z|^{p+n} .
$$

From Lemma 1, we see that the max in (4.5) occurs when $n=1$. This completes the proof of Theorem 5 .

Theorem 10 . If $f(z)$ is in the class $R^{p}[\alpha, \beta]$, then

$$
\begin{gathered}
p|z|^{p-1}-\frac{(p+1)(p-\beta)}{2(1+p-\beta)(p-\alpha)}|z|^{p} \leq\left|f^{\prime}(z)\right| \leq p|z|^{p-1}+\frac{(p+1)(p-\beta)}{2(1+p-\beta)(p-\alpha)}|z|^{p} \\
(z \in U) .
\end{gathered}
$$

Equality holds for $f_{p+1}(z)$ given by (4.4).

Proof. We know that

$p|z|^{p-1}-\max _{n \in N} \frac{(p-\beta)(n+p)|z|^{n+p-1}}{(n+p-\beta) G^{p}(\alpha, n+1)} \leq\left|f^{\prime}(z)\right| \leq p|z|^{p-1}+\max _{n \in N} \frac{(p-\beta)(n+p)|z|^{n+p-1}}{(n+p-\beta) G^{p}(\alpha, n+1)}(z \in U)$.

From Lemma 1, we see that the max in (4.7) occurs when $n=1$. This completes the proof of Theorem 6 .

Theorem 11 .If $f(z)$ is in the class $C^{p}[\alpha, \beta]$, then 


$$
|f(z)| \geq|z|^{p}-\frac{(p-\beta)}{2\left(\frac{p+1}{p}\right)(1+p-\beta)(p-\alpha)}|z|^{p+1}
$$

and

$$
|f(z)| \leq|z|^{p}+\frac{(p-\beta)}{2\left(\frac{p+1}{p}\right)(1+p-\beta)(p-\alpha)}|z|^{p+1}
$$

for $z \in U$. The results are sharp for the function $f(z)$ given by

$$
f(z)=z^{p}-\frac{(p-\beta)}{2\left(\frac{p+1}{p}\right)(1+p-\beta)(p-\alpha)} z^{p+1} \quad(z \in U) .
$$

Proof. From Theorem 4, we have that

$$
|f(z)| \geq|z|^{p}-\max _{n \in N} \frac{(p-\beta)}{\left(\frac{p+n}{p}\right)(n+p-\beta) G^{p}(\alpha, n+1)}|z|^{p+n}
$$

and

$$
|f(z)| \leq|z|^{p}+\max _{n \in N} \frac{(p-\beta)}{\left(\frac{p+n}{p}\right)(n+p-\beta) G^{p}(\alpha, n+1)}|z|^{p+n}
$$

for $z \in U$. From Lemma 1, we see that the max in (4.11) and (4.12) occur when $n=1$. This completes the proof of Theorem 7 .

Corollary 12 .If $f(z)$ is in the class $C^{p}[\alpha, \beta]$. Then $f(z)$ is included in a disc with its center at the origin and radius $r$ given by

$$
r=1+\frac{(p-\beta)}{2\left(\frac{p+1}{p}\right)(1+p-\beta)(p-\alpha)} .
$$

Theorem 13 .If $f(z)$ is in the class $C^{p}[\alpha, \beta]$, then

$$
\left|f^{\prime}(z)\right| \geq p|z|^{p-1}-\frac{p(p-\beta)}{2(1+p-\beta)(p-\alpha)}|z|^{p}
$$

and

$$
\left|f^{\prime}(z)\right| \leq p|z|^{p-1}+\frac{p(p-\beta)}{2(1+p-\beta)(p-\alpha)}|z|^{p}
$$

for $z \in U$. The bounds for (4.14) and (4.15) are sharp for the function $f(z)$ given by (4.10). 
Proof. By means of Theorem 4, we note that

$$
\left|f^{\prime}(z)\right| \geq p|z|^{p-1}-\max _{n \in N} \frac{p(p-\beta)}{(n+p-\beta) G^{p}(\alpha, n+1)}|z|^{p+n-1}
$$

and

$$
\left|f^{\prime}(z)\right| \leq p|z|^{p-1}+\max _{n \in N} \frac{p(p-\beta)}{(n+p-\beta) G^{p}(\alpha, n+1)}|z|^{p+n-1} .
$$

Also by using Lemma 1, we see that the max in (4.16) and (4.17) occur when $n=1$. This completes the proof of Theorem 8 .

Remark 1 . Making use of the relationship (1.15) between the classes $R^{p}[\alpha, \beta]$ and $C^{p}[\alpha, \beta]$, we can deduce Theorem 8 from Theorem 5.

\section{$5 \quad$ Integral operators}

Theorem 14 . Let the function $f(z)$ defined by (1.11) be in the class $R^{p}[\alpha, \beta]$, and let $c$ be a real number such that $c>-p$. Then the function $F(z)$ defined by

$$
F(z)=\frac{c+p}{z^{c}} \int_{0}^{z} t^{c-1} f(t) d t
$$

also belongs to the class $R^{p}[\alpha, \beta]$.

Proof. From the representation of $F(z)$, it follows that

$$
F(z)=z^{p}-\sum_{n=1}^{\infty} b_{p+n} z^{p+n}
$$

where

$$
b_{p+n}=\left(\frac{c+p}{c+p+n}\right) a_{p+n}
$$

Therefore

$$
\begin{aligned}
& \qquad \sum_{n=1}^{\infty}(n+p-\beta) G^{p}(\alpha, n+1) b_{p+n}=\sum_{n=1}^{\infty}(n+p-\beta) G^{p}(\alpha, n+1)\left(\frac{c+p}{c+p+n}\right) a_{p+n} \\
& \quad \leq \sum_{n=1}^{\infty}(n+p-\beta) G^{p}(\alpha, n+1) a_{p+n} \leq(p-\beta), \\
& \text { since } f(z) \in R^{p}[\alpha, \beta] \text {. Hence, by Theorem } 1, F(z) \in R^{p}[\alpha, \beta] .
\end{aligned}
$$


Corollary 15 . Under the same conditions as Theorem 9, a similar proof shows that the function $F(z)$ defined by (5.1) is in the class $C^{p}[\alpha, \beta]$, whenever $f(z)$ is in the class $C^{p}[\alpha, \beta]$.

\section{Radius of starlikeness}

Since $f(z)$ defind by $(1.11)$ is p-valent in the unit $\operatorname{disc} U$ if

$$
\sum_{n=1}^{\infty}\left(\frac{p+n}{p}\right) a_{p+n} \leq 1 \quad(c f .[4])
$$

we can see that $R^{p}[\alpha, \beta]$ is a subclass of $T(p)$ if $0 \leq \alpha \leq \frac{2 p-1}{2}, 0 \leq \beta<$ $p, p \in N$ with the aid of Theorem 1. Also we can see that $C^{p}[\alpha, \beta]$ is a subclass of $T(p)$ if $0 \leq \alpha \leq \frac{(2 p-1)(p-\beta)+2 p}{2(1+p-\beta)}, 0 \leq \beta<p, p \in N$ with the aid of Theorem 2.

Theorem 16 . Let the function $f(z)$ defined by (1.11) be in the class $R^{p}[\alpha, \beta]$.

Then $f(z)$ is p-valently starlike of order $\delta(0 \leq \delta<p)$ in $|z|<r_{1}$, where

$$
r_{1}=\inf _{n \in N}\left\{\frac{(p-\delta)(n+p-\beta) G^{p}(\alpha, n+1)}{(n+p-\delta)(p-\beta)}\right\}^{\frac{1}{n}}(n \geq 1) .
$$

The result is sharp, with the extremal function $f(z)$ given by (2.5).

Proof. It is sufficient to show that $\left|\frac{z f^{\prime}(z)}{f(z)}-p\right| \leq p-\delta$ for $|z|<r_{1}$. We have

$$
\left|\frac{z f^{\prime}(z)}{f(z)}-p\right| \leq \frac{\sum_{n=1}^{\infty} n a_{p+n}|z|^{n}}{1-\sum_{n=1}^{\infty} a_{p+n}|z|^{n}}
$$

Thus $\left|\frac{z f^{\prime}(z)}{f(z)}-p\right| \leq p-\delta$ if

$$
\sum_{n=1}^{\infty} \frac{(n+p-\delta)}{(p-\delta)} a_{p+n}|z|^{n} \leq 1
$$

Hence, by Theorem 1, (6.3) will be true if

$$
\frac{(n+p-\delta)}{(p-\delta)}|z|^{n} \leq \frac{(n+p-\beta) G^{p}(\alpha, n+1)}{(p-\beta)}
$$


or if

$$
|z| \leq\left\{\frac{(p-\delta)(n+p-\beta) G^{p}(\alpha, n+1)}{(n+p-\delta)(p-\beta)}\right\}^{\frac{1}{n}}(n \geq 1) .
$$

The theorem follows easily from (6.4).

Corollary 17 . Let the function $f(z)$ defined by (1.11) be in the class $R^{p}[\alpha, \beta]$. Then $f(z)$ is p-valently convex of order $\delta(0 \leq \delta<p)$ in $|z|<r_{2}$, where

$$
r_{2}=\inf _{n}\left\{\frac{p(p-\delta)(n+p-\beta) G^{p}(\alpha, n+1)}{(n+p)(n+p-\delta)(p-\beta)}\right\}^{\frac{1}{n}}(n \geq 1) .
$$

The result is sharp, with the extremal function $f(z)$ given by $(2.5)$.

Theorem 18 . Let the function $f(z)$ defined by (1.11) be in the class $C^{p}[\alpha, \beta]$, $0 \leq \alpha \leq \frac{(2 p-1)(p-\beta)+2 p}{2(1+p-\beta)}, 0 \leq \beta<p$ and $p \in N$. Then $f(z)$ is $p$ valently starlike of order $\delta(0 \leq \delta<p)$ in $|z|<r_{3}$, where

$$
r_{3}=\inf _{n \in N}\left\{\frac{(p-\delta)(p+n)(n+p-\beta) G^{p}(\alpha, n+1)}{p(p-\beta)(n+p-\delta)}\right\}^{\frac{1}{n}} .
$$

The result is sharp, with the extremal function $f(z)$ given by

$$
f(z)=z^{p}-\frac{(p-\beta)}{\left(\frac{p+n}{p}\right)(n+p-\beta) G^{p}(\alpha, n+1)} z^{p+n}(p, n \in N) .
$$

Corollary 19 . Let the function $f(z)$ defined by $(1.11)$ be in the class $C^{p}[\alpha, \beta]$, $0 \leq \alpha \leq \frac{(2 p-1)(p-\beta)+2 p}{2(1+p-\beta)}, 0 \leq \beta<p$ and $p \in N$. Then $f(z)$ is $p$ valently convex of order $\delta(0 \leq \delta<p)$ in $|z|<r_{4}$, where

$$
r_{4}=\inf _{n \in N}\left\{\frac{(p-\delta)(n+p-\beta) G^{p}(\alpha, n+1)}{(p-\beta)(n+p-\delta)}\right\}^{\frac{1}{n}}(n \geq 1) .
$$

The result is sharp, with the extremal function $f(z)$ given by $(6.7)$. 


\section{Modified Hadamard products}

Let the functions $f_{j}(z)(j=1,2)$ be defined by (3.1). The modified Hadamard product of $f_{1}(z)$ and $f_{2}(z)$ is defined by

$$
\left(f_{1} * f_{2}\right)(z)=z^{p}-\sum_{n=1}^{\infty} a_{p+n, 1} a_{p+n, 2} z^{p+n} .
$$

Theorem 20 . Let the functions $f_{j}(z)(j=1,2)$ defined by (3.1) be in the class $R^{p}[\alpha, \beta]$. Then $\left(f_{1} * f_{2}\right)(z) \in R^{p}[\alpha, \gamma(\alpha, \beta, p)]$, where

$$
\gamma(\alpha, \beta, p)=p-\frac{(p-\beta)^{2}}{2(1+p-\beta)^{2}(p-\alpha)-(p-\beta)^{2}} .
$$

The result is sharp.

Proof. . Employing the technique used earlier by Schild and Silverman [11], we need to find the largest $\gamma=\gamma(\alpha, \beta, p)$ such that

$$
\sum_{n=1}^{\infty} \frac{(n+p-\gamma) G^{p}(\alpha, n+1)}{(p-\gamma)} a_{p+n, 1} a_{p+n, 2} \leq 1
$$

Since

$$
\sum_{n=1}^{\infty} \frac{(n+p-\beta) G^{p}(\alpha, n+1)}{(p-\beta)} a_{p+n, 1} \leq 1
$$

and

$$
\sum_{n=1}^{\infty} \frac{(n+p-\beta) G^{p}(\alpha, n+1)}{(p-\beta)} a_{p+n, 2} \leq 1,
$$

by the Cauchy - Schwarz inequality we have

$$
\sum_{n=1}^{\infty} \frac{(n+p-\beta) G^{p}(\alpha, n+1)}{(p-\beta)} \sqrt{a_{p+n, 1} a_{p+n, 2}} \leq 1 .
$$

Thus it is sufficient to show that

$$
\begin{gathered}
\frac{(n+p-\gamma) G^{p}(\alpha, n+1)}{(p-\gamma)} a_{p+n, 1} a_{p+n, 2} \leq \frac{(n+p-\beta) G^{p}(\alpha, n+1)}{(p-\beta)} \sqrt{a_{p+n, 1} a_{p+n, 2}} \\
(n \geq 1), \quad
\end{gathered}
$$

that is, that 


$$
\sqrt{a_{p+n, 1} a_{p+n, 2}} \leq \frac{(n+p-\beta)(p-\gamma)}{(n+p-\gamma)(p-\beta)}
$$

Note that

$$
\sqrt{a_{p+n, 1} a_{p+n, 2}} \leq \frac{(p-\beta)}{(n+p-\beta) G^{p}(\alpha, n+1)}(n \geq 1) .
$$

Consequently, we need only to prove that

$$
\frac{(p-\beta)}{(n+p-\beta) G^{p}(\alpha, n+1)} \leq \frac{(n+p-\beta)(p-\gamma)}{(n+p-\gamma)(p-\beta)}(n \geq 1)
$$

or, equivalently, that

$$
\gamma \leq p-\frac{n(p-\beta)^{2}}{(n+p-\beta)^{2} G^{p}(\alpha, n+1)-(p-\beta)^{2}}(n \geq 1) .
$$

Since

$$
A(n)=p-\frac{n(p-\beta)^{2}}{(n+p-\beta)^{2} G^{p}(\alpha, n+1)-(p-\beta)^{2}}
$$

is an increasing function of $n(n \geq 1)$ for $0 \leq \alpha \leq \frac{2 p-1}{2}, 0 \leq \beta<p$ and $p \in$ $N$, letting $n=1$ in (7.12), we obtain

$$
\gamma \leq A(1)=p-\frac{(p-\beta)^{2}}{2(1+p-\beta)^{2}(p-\alpha)-(p-\beta)^{2}},
$$

which completes the proof of Theorem 12 .

Finally, by taking the functions

$$
f_{j}(z)=z^{p}-\frac{(p-\beta)}{2(1+p-\beta)(p-\alpha)} z^{p+1}(j=1,2)
$$

we can see that the result is sharp.

Corollary 21 . For $f_{j}(z)(j=1,2)$ as in Theorem 12, we have

$$
h(z)=z^{p}-\sum_{n=1}^{\infty} \sqrt{a_{p+n, 1} a_{p+n, 2}} z^{p+n}
$$

belongs to the class $R^{p}[\alpha, \beta]$.

The result follows from the inequality (7.6). It is sharp for the same functions as in Theorem 12. 
Corollary 22 . Let the functions $f_{j}(z)(j=1,2)$ defined by $(3.1)$ be in the class $C^{p}[\alpha, \beta]$. Then $\left(f_{1} * f_{2}\right)(z) \in C^{p}[\alpha, \lambda(\alpha, \beta, p)]$, where

$$
\lambda(\alpha, \beta, p)=p-\frac{(p-\beta)^{2}}{2\left(\frac{p+1}{p}\right)(1+p-\beta)^{2}(p-\alpha)-(p-\beta)^{2}} .
$$

The result is sharp for the functions

$$
f_{j}(z)=z^{p}-\frac{(p-\beta)}{2\left(\frac{p+1}{p}\right)(1+p-\beta)(p-\alpha)} z^{p+1}(j=1,2) .
$$

\section{References}

[1] M. K. Aouf, H. M. Hossen and H. M. Srivastava, Certain subclasses of prestarlike functions with negative coefficients, Kumamoto J.Math.11 (1998), $1-17$.

[2] M. K. Aouf and G. S. Salagean, Certain subclasses of prestarlike functions with negative coeffcients, Studia Univ. Babes - Bolyia Math. 39 (1994), no.1, 19 - 30.

[3] G. A.Kumar and Gl. Reddy, Certain class of prestarlike functions, J. Math. Res. Exposition 12(1992), no.3, 402 -412.

[4] Ming-Po Chen, Multivalent functions with negative coefficoents in the unit disc, Tamkang J. Math. 17(1986), no.3,127-137.

[5] S. Owa, On certain classes of p-valent functions with negative coefficients, Simon Stevin, 59 (1985), 385 - 402.

[6] S. Owa and M.A. Al-Bassam, An application of the fractional calculus, Pure Appl. Math. Sci. 24(1986), no. 1 -2, 1 - 7.

[7] S. Owa and B.A. Uralegaddi, A class of functions $\alpha$-prestarlike of order $\beta$, Bull. Korean Math. Soc. 21(1984), no, 77 - 85.

[8] D.A. Patil and N.K.Thakare, On convex hulls and extreme points of pvalent starlike and convex classes with applications, Bull. Math. Soc. Sci. Math. R.S. Roumanie (N.S.), 27 (1983), no. 75, 145 - 160.

[9] R. K. Raina and H.M. Srivastava, A unified presentation of certain subclasses of prestarlike functions with negative coefficients, Comput. Math. Appl. 38 (1999), 71 - 78. 
[10] St. Ruscheweyh, Linear operators between classes of prestarlike functions, Comm. Math. Helv. 52 (1977) , 497-502.

[11] A. Schild and H. Silverman , Convolution of univalent functions with negative coefficients, Ann. Univ. Mariae Curie - Sklodowska Sect. A, 29 (1975), 99-106.

[12] T. Sheil - Small, H. Silverman and E. M. Silvia, Convolution multipliers and starlike functions, J. Analyse Math. 41 (1982), 181 - 192.

[13] G. M. Shenen, T. Q. Salim and M. S.Marouf, A certain class of multivalent prestarlike functions involving the Srivastava -Saigo - Owa fractional integral operator, Kyungpook Math. J. 44(2004), 353 - 362.

[14] H. Silverman and E. M. Silvia, Prestarlike functions with negative coefficients, Internat. J. Math. Math. Sci. 21(1979), no.3, 427 - 439.

[15] H. Silverman and E.M .Silvia, Subclasses of prestarlike functions, Math. Japon. 29(1984), 929 - 936.

[16] H. M. Srivastava and M. K. Aouf, Some applications of fractional calculus operators to certain subclasse of prestarlike functions with negative coefficients, Comput. Math. Appl. 30 (1995), no.1, 53 - 61.

[17] B. A. Uralegaddi and S. M. Sarangi, Certain generalization of prestarlike functions with negative coefficients, Ganita 34 (1983), 99 - 105.

Received: July 11, 2006 